\title{
La biopsie liquide
}

Une voie possible pour

le dépistage du cancer

Alain R. Thierry ${ }^{1-4}$, Rita Tanos ${ }^{1-4}$

> La biopsie liquide est apparue comme une voie prometteuse pour le dépistage du cancer. En effet, plusieurs biomarqueurs comme les ADN circulants, les cellules tumorales circulantes, les micro-ARN circulants etc. se sont révélés prometteurs pour le théragnostic ou le suivi du patient. La détection précoce peut aider à réduire la mortalité associée au cancer et augmenter la survie globale des patients. La plupart des types de cancer manquent de biomarqueurs spécifiques et le développement de techniques de dépistage efficaces appliquées en clinique a été limité malgré des efforts intenses dans ce domaine. La nature non invasive de la biopsie liquide lui donne un avantage vis-à-vis d'autres méthodes, notamment pour le développement de tests de dépistage du cancer. Les différentes études fondées sur l'analyse de la biopsie liquide dans le but de développer des tests de dépistage et de détection précoce du cancer sont présentées dans cette revue. Bien qu'actuellement aucun test développé à partir de la biopsie liquide s'avère à la fois assez spécifique et sensible pour être utilisé comme test universel de dépistage, le potentiel de cette nouvelle approche apparaît de plus en plus crédible, eu égard aux récents développements de méthodes sophistiquées, notamment multiparamétriques. <

\section{Le dépistage du cancer}

La progression du cancer à des stades tardifs sans apparition de symptômes est l'une des principales raisons pour lesquelles il figure parmi les premières causes de décès dans le monde. Le dépistage par sa détection précoce, avant qu'une personne présente des signes de la maladie et lorsque le traitement est plus efficace, augmenterait les chances de rétablissement et la survie

Vignette (Photo @ Daniel Birnbaum).

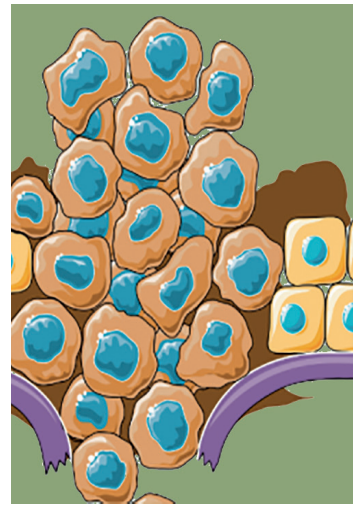

${ }^{1}$ IRCM, Institut de recherche en cancérologie de Montpellier, 208, avenue des Apothicaires, Montpellier, F-34298, France. ${ }^{2}$ Inserm U1194, Montpellier, F-34298, France.

${ }^{3}$ Université de Montpellier, Montpellier, F-34090, France. ${ }^{4}$ Institut régional du cancer de Montpellier, Montpellier, F-34298, France.

alain.thierry@inserm.fr

globale des patients. II pourrait ainsi aider à réduire la mortalité liée au cancer [1].

Un test diagnostique diffère d'un test de dépistage par le fait qu'il est utilisé lorsqu'un sujet présente des signes ou des symptômes afin de déterminer la présence ou l'absence d'une maladie et qu'il est habituellement pratiqué après un test de dépistage positif pour établir un diagnostic définitif. Le développement de techniques de dépistage pour la détection précoce ne s'est pas avéré efficace pour de nombreux types de cancers, notamment en ce qui concerne la prévention de la mortalité liée à la maladie. Cela est dû à diverses raisons techniques et cliniques, la relative faible performance des tests en termes de sensibilité et de spécificité, l'hétérogénéité inter et intra-tumorale, ou à des facteurs épidémiologiques.

\section{Comment évaluer un test de dépistage du cancer}

En 1968, l'Organisation mondiale de la santé (OMS) a publié des directives, appelées souvent critères de Wilson [2], sur les principes et la pratique du dépistage de la maladie. En 2008, avec l'émergence de nouvelles technologies génomiques, l'Organisation a modifié ces directives avec la définition suivante [3] : « Le programme de dépistage doit répondre à un besoin reconnu, et ses objectifs doivent être définis dès le départ. Il devrait y avoir une population cible définie et des preuves scientifiques de l'efficacité du programme de dépistage. Le programme devrait intégrer l'éducation, les tests, les services cliniques et la gestion de programme, ainsi que l'assurance de la qualité et des mécanismes pour minimiser les risques potentiels de dépistage. Il devrait garantir un choix informé, la confidentialité et le respect de l'autonomie, et promouvoir l'équité et l'accès au dépistage pour l'ensemble de la population cible. L'évaluation devrait être planifiée dès le départ et les avantages globaux du dépistage devraient l'emporter sur les dommages ». 
Un test de dépistage idéal du cancer permettrait donc d'établir une distinction parfaite entre les personnes atteintes et indemnes [1]. En pratique, un test de dépistage présente un certain pourcentage de faux positifs (des personnes détectées positives par le test alors qu'elles ne sont pas malades) et de faux négatifs (des personnes malades non détectées). La détermination de ces valeurs est soigneusement prise en compte lors de l'évaluation des avantages et des inconvénients du test, en considérant le rapport bénéfices/risques. Une détection précoce ne conduit pas toujours à de meilleurs résultats. Elle révèle parfois des faux positifs, ce qui crée des inquiétudes chez les personnes détectées et peut entraîner un traitement inutile. Le sur-diagnostic est ainsi remis en question, notamment pour le dépistage du cancer du sein. Le but du dépistage, quel que soit sa performance, doit consister à proposer uniquement un avantage pour la santé de l'individu.

Lorsqu'un nouveau test est développé, il est régulièrement comparé au test de référence, c'est-à-dire «le meilleur test disponible » qui consiste généralement en un test de diagnostic considéré comme définitif, comme une biopsie par exemple. Cette dernière est souvent coûteuse, invasive et potentiellement dangereuse, trop tardive ou peu pratique, pour être largement utilisée comme test de dépistage [1]. Un nouveau test devrait donc être non invasif, ou moins coûteux ou plus bénéfique, le rapport coûts/bénéfices étant au centre de l'évaluation par les administrations de santé publique.

La validité d'un test se traduit par une sensibilité (Se) élevée, qui est la capacité du test à identifier correctement les personnes qui ont la maladie, et une haute spécificité ( $\mathrm{Sp}$ ), reflétant sa capacité à identifier correctement celles qui ne sont pas atteintes. La courbe ROC (receiver operating characteristic curve) combine ces deux paramètres en représentant la Se en ordonnée et le complément de la Sp (ou 1-Sp) en abscisse. Elle met donc en rapport la proportion de vrais positifs (parmi les malades) avec la proportion de faux positifs (parmi les non-malades) pour toutes les valeurs-seuil du test [4]. L'aire sous la courbe ROC (AUC pour area under curve) est un indice simple et quantitatif de la performance du test et révèle sa capacité à discriminer entre malades et non-malades. Un test parfait présente une AUC égale à 1 ; pour un test sans valeur discriminante, l'aire sous la courbe sera de 0,5 .

Une attention toute particulière doit être apportée aux cohortes des individus testés afin d'évaluer les performances d'un test de dépistage. Une étude en aveugle et une analyse intégrant des individus déjà diagnostiqués, ainsi que des personnes avant diagnostic dans des populations à risques, sont une nécessité.

\section{Biomarqueurs et tests de dépistage conventionnels}

Différents tests de dépistage et biomarqueurs sont utilisés en pratique pour identifier divers types de cancer (Tableau I). Le test de frottis (pap smear test) est par exemple utilisé pour la dysplasie cervicale, ou le cancer du col de l'utérus. II a une haute spécificité mais ne montre pas une sensibilité élevée [5]. La mammographie est le test le plus couramment utilisé pour le dépistage du cancer du sein. Ce test a joué un rôle clé dans la réduction de la mortalité par ce cancer, mais il présente une sensibilité limitée ainsi que de nombreux faux positifs et la possibilité de sur-diagnostic, d'où des opinions récentes assez contradictoires quant à son utilisation à une échelle de masse. Le dosage des marqueurs sériques, comme l'antigène tumoral CA 15-3 (cancer antigen 15-3), a également été appliqué pour le cancer du sein. Bien que très spécifique (Sp de $98 \%$ ), il a cependant montré une sensibilité moyenne (Se de $55,6 \%$ ) [6] ; il est donc préférentiellement utilisé pour le suivi de la réponse au traitement plutôt que pour le dépistage et le diagnostic précoce. Le test de détection de l'antigène prostatique spécifique (prostate specific antigen, PSA) aide à révéler un cancer de la prostate avant l'apparition des symptômes [7]. Un taux élevé de PSA ne résulte pas toujours de la présence d'un cancer et une polémique autour du dosage du PSA et le dépistage du cancer de la prostate est apparue en 2009. Deux études, une américaine [8] et une européenne [9], publiées dans le New England Journal of Medicine ont montré des résultats contradictoires sur la capacité de ce test de réduire le taux de mortalité ; cependant, en 2017, une ré-évaluation statistique des résultats de ces deux études a confirmé la diminution de la mortalité dans le groupe avec dépistage [10]. La tomodensitométrie à faible dose (low-dose CT scan) est généralement recommandée pour les personnes à risque élevé de développer un cancer du poumon, mais certaines tumeurs pourraient ne pas être détectées et d'autres pourraient se développer entre le dépistage et la détection. Pour le cancer colorectal (CCR), les deux glycoprotéines sériques les plus communément utilisées comme biomarqueurs, le CEA (carcinoembryonic antigen) et le CA19-9 (carbohydrate antigen 19-9), ne sont pas appropriées pour son dépistage en raison de leur faible sensibilité et spécificité, surtout pour les stades précoces (Se entre $40,9 \%$ et $51,8 \%$, Sp entre $85,2 \%$ et $95 \%$, pour le CEA [11]). Leurs mesures sont en fait plus appropriées pour le suivi de la récurrence ou de la réponse au traitement. D'autres tests de dépistage du CCR, fondés sur des analyses de selles par exemple, ont donc été développés. Le hemoccult fecal occult blood test (FOBT) est utilisé depuis longtemps pour détecter du sang dans les échantillons de selles comme indicateur précoce du CCR avec une sensibilité variant entre 12,9\% et $79,4 \%$ et une spécificité de $86,7 \%$ à $97,7 \%$ [12]. Ce test présentant certains inconvénients, le fecal immunochemical test (FIT) est plus couramment utilisé en raison de son faible coût et de sa sensibilité globale de $79 \%$ et sa spécificité de $94 \%$ [12]. Le test de l'ADN fécal (fecal DNA test) [13] a permis la détection de cancers invasifs et d'adénomes avec une dysplasie de haut grade, avec une sensibilité de $40,8 \%$ et une spécificité 
de 94,4\% [14]. Cologuard a été le premier test d'ADN fécal commercialisé et approuvé par la Food and drug administration (FDA) en 2014 aux États-Unis. Son coût est plus élevé que les autres tests et sa spécificité inférieure, mais il présente une sensibilité plus élevée que le FIT, que ce soit pour le CCR ou la présence de polypes [15].

\section{La biopsie liquide}

Selon la définition du dictionnaire du NCl (National Cancer Institute), la biopsie liquide est "un test effectué sur un échantillon de sang pour la recherche de cellules cancéreuses ou de fragments d'ADN provenant d'une tumeur et circulant dans le sang » [16]. Cette définition est, selon nous, plutôt imparfaite. Réunir sous le même terme de «biopsie liquide » une entité si complexe que sont des cellules et des macromolécules (ADN, ARN ou microARN) ne peut dépendre que de la nature liquide de leur prélèvement, de leur origine tumorale, et de l'information diagnostique qu'elles peuvent fournir en oncologie clinique. Comme indiqué dans le dictionnaire du $\mathrm{NCl}$, «une biopsie liquide peut être utilisée pour aider à trouver un cancer à un stade précoce, elle peut également être utilisée pour planifier un traitement ou déterminer si le traitement est efficace ou si le cancer a resurgi. Ainsi que permettre plusieurs prélèvements sanguins au fil du temps peut aussi aider les médecins à comprendre les changements moléculaires qui se produisent dans une tumeur ». Malgré l'incohérence du terme regroupant ces différentes entités, nous utiliserons dans cette revue le terme «biopsie liquide » selon le dictionnaire du $\mathrm{NCl}$ et son utilisation conventionnelle dans la littérature. La terminologie biopsie liquide ne s'applique donc principalement qu'en oncologie, en miroir de la biopsie de tissu tumoral. Elle ne peut s'appliquer aux ADN circulants que l'on analyse dans d'autres domaines : diagnostic prénatal, inflammations sévères/aiguës (sepsis), transplantation, ou sport.

Les biopsies liquides ne sont pas limitées au sang. L'urine, la salive ou le liquide cervical peuvent également être utilisés, puisque l'information génétique est également présente dans ces fluides. La recherche sur les biopsies liquides ne s'est développée qu'au cours de la dernière décennie, générant un intérêt croissant en oncologie, et très récemment pour le dépistage ou la détection précoce du cancer.

\section{L’ADN circulant}

Pour la première fois, il y a plus de 70 ans, des chercheurs ont montré que les tumeurs libéraient des acides nucléiques dans les fluides corporels [17]. Plus récemment, les ADN circulants (ou ADNcir, pour circulating cell free DNA) se sont avérés porter une partie de la même information moléculaire que les biopsies tissulaires [18, 19]. L'ADNcir est ainsi apparu comme un biomarqueur potentiel particulièrement dans le cancer et il fait l'objet d'études en recherche translationnelle et clinique [19]. Ce biomarqueur permettrait de diagnostiquer, de surveiller la récurrence et d'évaluer la réponse à la thérapie, uniquement par un prélèvement sanguin. Une analyse qualitative de l'ADNcir permettrait, par exemple, la détection non invasive d'altérations géniques liées à différents types de cancer, comme les mutations touchant les gènes KRAS (Kirsten rat sarcoma viral oncogene homolog) dans le cancer colorectal, et EGFR (epidermal growth factor receptor) dans le cancer des poumons. Une analyse quantitative de son taux circulant pourrait renseigner sur la présence ou l'absence d'un cancer, suivre le développement de la tumeur ou être le signe d'une éventuelle rechute. Le profil de méthylation de I'ADNcir est également étudié afin de déterminer le tissu dont il est originaire, ainsi que sa taille et sa fragmentation, pour caractériser sa structure et ses mécanismes de libération. Plusieurs limites de l'analyse de l'ADNcir réduisent l'intérêt de son utilisation en clinique. L'une des contraintes majeures de ce type de détection est certainement le manque de procédure standardisée (SOP, pour standard operating procedure), notamment en ce qui concerne le contrôle de la phase pré-analytique, en particulier le contrôle des différents paramètres intervenant depuis le prélèvement sanguin jusqu'à l'analyse de l'ADNcir [20]. Une grande majorité des méthodes de détection et d'analyse de I'ADNcir ne se sont pas avérées assez sensibles en raison de la rareté des ADNcir mutés présents dans l'échantillon: une cellule tumorale portant la mutation ne libère qu'un seul génome dans la circulation et donc qu'un (ou très rarement deux) allèle muté ; les ADNcir mutés peuvent être en très grande minorité, compte tenu de la fréquente disproportion entre cellules malignes (mutées) et cellules du microenvironnement tumoral (cellules du stroma, endothéliales et lymphocytaires). Toutefois, les efforts de développement technologique récents permettent d'entrevoir des solutions envisageables cliniquement. Les administrations européennes de santé ont déjà autorisé en oncologie thoracique, l'analyse de l'ADN tumoral circulant (mutations du gène EGFR) comme une alternative à la biopsie tissulaire, uniquement lorsque celle-ci est impossible à réaliser ou insuffisante. D'autres tests compagnons aux thérapies ciblées utilisant l'examen de l'ADNcir pourraient donc être autorisés prochainement.

Le potentiel de ce biomarqueur pour le dépistage précoce du cancer et des altérations qualitatives et quantitatives de cet ADN a fait l'objet de nombreuses études [21]. Cependant, malgré cette recherche intense, peu de tests ont été implémentés en pratique clinique. Des données contradictoires concernant la concentration totale d'ADNcir nucléaire ont en effet limité la mise au point et l'utilisation de ces tests en clinique : alors que les concentrations plasmatiques d'ADNcir chez les patients cancéreux varient de quelques ng à plusieurs milliers par millilitre, un chevauchement avec la concentration mesurée chez les individus sains a été retrouvé $[21,22]$. 
Un test de dépistage fondé sur l'ADNcir devrait permettre de distinguer les processus non cancéreux et précancéreux de ceux de la malignité invasive afin d'atteindre une sensibilité clinique élevée. L'utilité d'un tel test résulterait également de sa capacité de fournir des informations sur l'origine tissulaire de l'ADNcir, ce qui devrait être possible étant donné les différences d'altérations somatiques existant entre les différents types de tumeurs.

Jusqu'à présent, plusieurs groupes ont travaillé sur le développement de tests pour le dépistage et le diagnostic précoce de plusieurs types de cancer à partir d'une seule analyse de sang (Tableau I). Ainsi, pour le dépistage du cancer colorectal par exemple, plusieurs études ont porté sur la détection dans le plasma du gène méthylé de la Septine 9 , une protéine de la famille des protéines liant le GTP (GTP-binding protein) dont le taux est significativement plus élevé chez les patients atteints de cancer colorectal par rapport à celui observé chez les individus sains, ce qui en fait un biomarqueur potentiel de ce cancer [23]. L'Epi proColon ${ }^{\circledR}$ (Epigenomics AG Corporation, Berlin, Allemagne), fondé sur la détection dans le sang par PCR (polymerase chain reaction) en temps réel du gène codant la Septine 9 méthylé est le seul test de dépistage sanguin de l'hyperméthylation d'ADN pour le CCR [24]. II montre jusqu'à présent les meilleurs résultats parmi les tests commerciaux de détection du cancer par analyse sanguine, permettant de distinguer les patients atteints de CCR des individus sains avec une sensibilité de 75-81\% et une spécificité de 96-99\%.

D'autres gènes ou régions de l'ADNcir ont également été examinés afin de déterminer la pertinence de leur détection pour un diagnostic précoce. Ainsi, différentes régions promotrices hyperméthylées de certains gènes identifiés comme spécifiques au cancer colorectal ont été examinées. Les AUC (aire sous la courbe ROC) les plus élevées $(0,85)$ ont été obtenues pour un test combinant la détection de sept promoteurs, après normalisation de l'âge et du sexe [25]. Une combinaison de quatre marqueurs de nucléosomes circulants a été développée par Volition et a fourni une AUC de 0,87 (0,97 pour le même panel avec l'ajustement de l'âge) pour discriminer patients atteints de CCR et témoins sains, avec une sensibilité élevée pour les stades précoces (75 et $86 \%$ respectivement pour les stades I et II, pour une spécificité de $90 \%$ ). Une deuxième combinaison de quatre autres biomarqueurs de nucléosomes circulants a fourni une AUC de 0,72 pour discriminer patients présentant des polypes et individus sains [26], montrant ainsi une application potentielle de ce test dans le dépistage du CCR. Le potentiel diagnostic de l'ADNcir et son taux sanguin ont également été examinés dans d'autres types de cancer. Ainsi, dans le cas du cancer du poumon, une analyse quantitative a montré des taux accrus d'ADNcir chez les patients cancéreux, comparés aux individus sains, avec une valeur d'AUC d'environ 0,88 [27]. Des résultats similaires ont été observés pour le cancer du sein avec $78 \%$ de sensibilité et $83 \%$ de spécificité [28], ainsi que le cancer de l'ovaire [29] avec une sensibilité de $70 \%$ et une spécificité de $90 \%$.

L'ADN circulant est constitué non seulement d'ADN d'origine nucléaire mais aussi d'ADN mitochondrial (ADNmt), dont certaines altérations ont été associées à un dysfonctionnement mitochondrial et au développement d'un cancer. La signification clinique des niveaux
d'ADN mitochondrial dans le sang périphérique et de son intégrité a été notamment étudiée pour différents types de cancers tels que le poumon, le sein, le cancer colorectal, le lymphome non hodgkinien $[30,31]$. Les données restent actuellement discordantes et aucune conclusion ne peut être donnée. L'absence d'études pré-analytiques et analytiques concernant I'ADN mitochondrial circulant, qui est mal caractérisé, notamment ses propriétés structurales, pourrait expliquer en partie ces discordances.

\section{Les cellules tumorales circulantes}

La remarquable découverte dans la circulation sanguine de cellules libérées ou s'échappant de la tumeur par des chercheurs australiens il y a plus d'un siècle [32] a été à l'origine d'intenses recherches au cours de la dernière décennie. Les cellules tumorales circulantes (CTC) sont cependant difficiles à isoler et ne sont pas toujours le reflet des cellules génétiquement cancéreuses. La valeur de ces CTC a également été évaluée dans plusieurs études pour le dépistage de différents types de cancers (Tableau I). Dans le cancer du poumon, Tanaka et al. ont montré que le dénombrement des CTC ne présentait pas un potentiel discriminant adéquat entre patients cancéreux et individus sains (avec une AUC de 0,598) [33]. D'autres groupes ont déterminé en revanche que la détection d'un nombre de CTC supérieur à 25 présentait une sensibilité ( $89 \%$ ) et une spécificité élevées (100\%) pour distinguer maladie bénigne et maligne [34]. Les CTC ont également été détectées chez des patients atteints de bronchopneumopathie chronique obstructive (BPCO), un facteur de risque du cancer du poumon, sans présenter de signes cliniques de cancer [35]. Wang et al. ont évalué la valeur des CTC pour le diagnostic précoce du cancer du poumon : une AUC de près de 0,80 ( $\mathrm{Se}=77,7 \%$ et $\mathrm{Sp}=89,5 \%$ ) [36] a été mesurée pour un seuil de 8,7 unités de CTC exprimant le récepteur du folate (ou vitamine B9) entre le groupe témoin et les patients atteints.

La technologie permettant d'isoler et de phénotyper les CTC a rapidement progressé. Néanmoins, la complexité de leur analyse et le faible signal analytique limitent leur utilité clinique par rapport aux méthodes fondées sur l'ADNcir tumoral [37]. En effet, lorsque les deux sont présents, les fragments d'ADNcir tumoral sont plus nombreux (d'un facteur de 50) que les CTC [37]. Ils apportent donc un signal analytique bien supérieur. Les CTC ne présentent en revanche pas le désavantage de I'ADNcir tumoral de devoir mesurer des quantités très faibles de fragments mutés dans le plasma, en raison 


\begin{tabular}{|c|c|c|c|c|c|c|c|}
\hline \multicolumn{2}{|c|}{ Catégorie } & Test & Cancer & Sensibilité & Spécificité & AUC & Réf \\
\hline \multirow{8}{*}{\multicolumn{2}{|c|}{$\begin{array}{c}\text { Biomarqueurs et tests } \\
\text { conventionnels } \\
\text { utilisés }\end{array}$}} & CEA & Colorectal & $41-52 \%$ & $85-95 \%$ & $0,63-0,77$ & [11] \\
\hline & & CA19-9 & $\begin{array}{l}\text { Colorectal/ } \\
\text { Pancréas }\end{array}$ & $23 \%$ & $95 \%$ & 0,64 & [11] \\
\hline & & FOBT & Colorectal & $13-79 \%$ & $87-98 \%$ & - & [12] \\
\hline & & $F I T$ & Colorectal & $79 \%$ & $94 \%$ & - & [12] \\
\hline & & $\begin{array}{c}\text { Cologuard } \\
\text { (test d'ADN fécal) }\end{array}$ & Colorectal & $41 \%$ & $94 \%$ & - & [13] \\
\hline & & CA 15-3 & Sein & $55,6 \%$ & $98 \%$ & - & [6] \\
\hline & & $\begin{array}{c}\text { PHI } \\
\text { (prostate health index) }\end{array}$ & Prostate & - & - & $0,69-0,77$ & {$[54]$} \\
\hline & & PSA & Prostate & - & - & 0,55 & {$[7]$} \\
\hline \multirow{12}{*}{$\begin{array}{l}\text { Biomarqueurs } \\
\text { circulants } \\
\text { en cours de } \\
\text { développement }\end{array}$} & & $\begin{array}{l}\text { Epi ProColon } \\
\text { (détection du gène de } \\
\text { la Septine } 9 \text { méthylé) }\end{array}$ & Colorectal & $75-81 \%$ & $96-99 \%$ & - & {$[23,24]$} \\
\hline & & 7 promoteurs méthylés & Colorectal & $91 \%$ & $73 \%$ & 0,85 & {$[25]$} \\
\hline & & $\begin{array}{c}\text { Volition } \\
\text { (marqueurs de } \\
\text { nucléosomes circulants) }\end{array}$ & Colorectal & $75 \%$ & $86-90 \%$ & 0,97 & [26] \\
\hline & $\begin{array}{c}\text { ADN } \\
\text { circulant }\end{array}$ & & Poumon & & & 0,88 & [27] \\
\hline & & Concentration totale & Sein & $78 \%$ & $83 \%$ & 0,91 & {$[28]$} \\
\hline & & d'ADNcir nucléaire & Ovaire & $70 \%$ & $90 \%$ & 0,89 & [29] \\
\hline & & & Colorectal & - & - & 0,91 & [22] \\
\hline & & $\begin{array}{l}\text { Détection du virus } \\
\text { d'Epstein-Barr }\end{array}$ & Nasopharynx & $97,1 \%$ & $98,6 \%$ & - & [49] \\
\hline & & CancerSeek & Divers & $30-99 \%$ & $99 \%$ & 0,91 & {$[48]$} \\
\hline & СTC & Numération & Poumon & $30-78 \%$ & $88-100 \%$ & $0,60-0,86$ & {$[33,34,36]$} \\
\hline & $\begin{array}{c}\text { miARN } \\
\text { circulants }\end{array}$ & Panel & $\begin{array}{l}\text { Sein } \\
\text { Poumon et } \\
\text { mésothélium }\end{array}$ & $73-75 \%$ & $54-79 \%$ & 0,67 & {$[41]$} \\
\hline & Autres & $\begin{array}{l}\text { Combinaison de } \\
\text { biomarqueurs } \\
\text { protéiques circulants }\end{array}$ & Sein & - & - & $0,70-0,99$ & {$[44]$} \\
\hline
\end{tabular}

Tableau I. Récapitulatif de différents tests de dépistage du cancer et de leurs performances actuelles. La sensibilité, la spécificité et l’AUC représentent les paramètres de performance.

de la libération importante d'ADNcir non muté issu majoritairement de l'ADN des cellules hématopoïétiques. Dans un essai récent réalisé sur des patients atteints d'un cancer du poumon, l'analyse de l'ADNcir tumoral a surpassé l'examen des CTC pour la détection de la mutation du gène KRAS, révélant des sensibilités respectives de $96 \%$ et de $52 \%$ [38]. 
Très récemment, un engouement certain s'est porté sur l'analyse de la cellule unique (single cell analysis). Techniquement réalisable, cette approche peut s'avérer d'importance pour le dépistage du cancer. Les études sont cependant peu nombreuses et les résultats peu convaincants pour l'instant [39]. Outre la paucité du nombre de CTC dans le sang, l'un des inconvénients majeurs de leur analyse est la nécessité de leur préparation immédiate juste après le prélèvement (dans la demi-journée), alors que le délai pour l'analyse d'ADNcir peut atteindre 5 jours si le sang est prélevé sur stabilisant. Les tests utilisant les CTC sont néanmoins plus liés à la terminologie de la biopsie liquide et, intrinsèquement, plus informatifs puisque la détermination des marqueurs cellulaires peut être combinée à l'information génétique dans un même échantillon.

\section{Les autres biomarqueurs circulants}

La pertinence diagnostique des micro-ARN circulants (miARN) dans le sang a été examinée chez des patients atteints de différents types de cancer (Tableau I). Dans le sang de patientes atteintes d'un cancer du sein, les miARN circulants associés aux tumeurs sont élevés et liés à la progression tumorale [40] et une signature multivariée de neuf miARN circulants a été validée et a permis de discriminer les patientes atteintes des femmes saines avec une AUC de 0,665 [41]. D’autres signatures de miARN circulants ont été identifiées pour le diagnostic précoce des tumeurs malignes pulmonaires [42].

La présence de macrophages circulants associés à la tumeur pourrait être utile comme outil de dépistage du cancer du sein et permettrait de différencier les patientes atteintes d'une maladie maligne de celles présentant une affection mammaire bénigne ou saines [43]. Dans ce cancer, la combinaison de plusieurs biomarqueurs circulants protéiques a aussi été évaluée et a montré des valeurs d'AUC variant entre 0,7 et 0,99 selon le sous-type de cancer [44].

Les plaquettes éduquées par la tumeur (tumor-educated platelets, $T \varepsilon P)$ sont un autre biomarqueur circulant. Les profils d'ARN messagers de ces TEP permettent en effet de révéler les patients cancéreux [45].

\section{Dépistage du cancer et analyse de l'ADN circulant : vers de nouvelles possibilités}

\section{Profil des altérations génétiques}

Les biopsies liquides ont le potentiel de permettre d'identifier les patients dont les tumeurs présentent des mutations spécifiques. Velculescu et al. ont récemment évalué les méthodes de séquençage de nouvelle génération (Next-Gen sequencing) appliquées à I'ADN circulant sur 138 patients atteints de tumeurs précoces. L'analyse a aussi été utilisée pour détecter la rechute après traitement. En utilisant un séquençage de type TEC-Seq (targeted error correction sequencing), le type de cancer a pu être identifié chez plus de la moitié des patients [46] : cinquante-huit gènes sont typiquement associés aux cancers du sein, du poumon, de l'ovaire et colorectal. Sur les 138 cancers examinés, 86 cancers de stade I et II ont été détectés et l'analyse des gènes, séquencés chez 100 patients, a montré les mêmes mutations dans les échantillons de sang et dans les échantillons de tissus tumoraux chez 82 patients. Aucun des patients sains n'a montré de mutations. Cette étude présente quelques limites avec la difficulté d'identifier l'ADN rare provenant de la tumeur et de révéler d'autres altérations génétiques ou mutations héréditaires ou somatiques. Des mutations du gène TP53 ont été retrouvées dans les échantillons sanguins de $49 \%$ des patients atteints du cancer de poumon à petites cellules (small cell lung cancer, SCLC) avec des formes alléliques significativement plus élevées chez les patients cancéreux [47].

Association du profil des altérations génétiques avec des biomarqueurs protéiques

En janvier 2018, un nouveau test sanguin pour le dépistage du cancer, appelé CancerSEદK, a reçu beaucoup d'attention [48]. L'un des éléments les plus innovants de l'étude réalisée sur le plasma de 1005 patients ayant reçu un diagnostic de cancer sans propagation au moment de l'inclusion et de 812 individus sains, était I'utilisation d'une combinaison de marqueurs génétiques et protéiques. CancerSદEK concernait le séquençage ciblé de seize gènes, associé à huit biomarqueurs protéiques caractéristiques d'un type spécifique de cancer, qui non seulement identifient la présence de cancers relativement précoces mais aussi contribuent à localiser l'organe d'origine de ces cancers. CancerS\&EK a permis de détecter $98 \%$ des cancers de l'ovaire et du foie, entre $60 \%$ et $70 \%$ des cancers de l'estomac, du pancréas, de l'œsophage, du côlon et du poumon, et $33 \%$ des cancers du sein. Bien que la performance de ce test sanguin reste actuellement inégalée, il présente des limitations dans un contexte de dépistage universel du cancer. CancerSEEK apparaît comme une confirmation d'un diagnostic au stade du pré-dépistage. Pour le qualifier de test de dépistage, il aurait dû être réalisé sur une population saine, avec potentiellement des patients asymptomatiques $(\rightarrow)$. Néanmoins, CancerskદK se montre intéressant pour la détection pré-

$(\rightarrow)$ Voir la Chronique de B. Jordan, $m / s n^{\circ} 4$, avril 2018, page 363

coce et le suivi des tumeurs à partir d'un échantillon de sang. Son développement est clairement un pas de plus vers un test de dépistage universel.

\section{Détection de génome viral}

Plutôt que d'analyser les cellules cancéreuses ellesmêmes, Lo et al. ont récemment décrit une étude visant à détecter le virus d'Epstein-Barr, impliqué dans la plupart des cas de cancer du nasopharynx, par la recherche de l'ADN viral libéré dans le sang par les cellules tumorales [49]. L'ADN viral a été retrouvé dans le sérum de 1112 individus d'une cohorte de 20000 hommes (soit 
$5,5 \%)$; 309 patients ont vu la confirmation de leur diagnostic un mois plus tard, et pour 34 d'entre eux, un cancer a été révélé après examen endoscopique et imagerie par résonance magnétique (IRM) ; une seule personne détectée négative au dépistage a développé un cancer en moins d'un an. Le test a détecté plus de cas positifs pour les stades les plus précoces de la maladie.

\section{Fragmentation de l'ADN circulant}

Nous avons observé que les fragments d'ADN courts étaient plus abondants dans le plasma de patients atteints de cancer colorectal que chez des individus sains. La quantité de fragments d'ADN circulants de moins de 145 paires de bases ( $p b$ ) est également directement liée à la concentration d'ADN tumoral circulant [50]. L'ADNcir muté provenant des cellules tumorales est en fait très fragmenté comparé à l'ADN cirulant non muté [22] : la détection de l'ADN tumoral circulant repose sur des amplicons inférieurs à 100 pb et l'analyse par microscopie à force atomique révèle des fragments d'ADNcir en moyenne de 135 pb chez les patients cancéreux [22]. Une forte discrimination entre cancers colorectaux de stade IV et individus sains a pu être observée en ciblant un petit amplicon (de $63 \mathrm{pb}$ ) [22]. Ces observations ont été confirmées par Leszinski et al. qui ont montré que l'ADNcir était significativement plus fragmenté chez les patients atteints de cancer colorectal que chez les individus sains ou atteints de maladies colorectales bénignes [51]. Plusieurs indices d'intégrité de l'ADN ont été évalués, avec de très diverses efficacités pour la discrimination des patients sains et cancéreux sans doute en raison du manque de procédures opératoires standards et du nombre de patients testés. Un pouvoir de dépistage avancé pour un indice d'intégrité d'ADN spécifique, déterminé par PCR (polymerase chain reaction) quantitative, a pu être obtenu. La stratégie utilisée est fondée sur la différence entre structure de l'ADNcir dérivant de cellules malignes et celle de l'ADNcir issu de cellules saines. Elle facilite la mise en œuvre de l'analyse et réduit les coûts des tests de dépistage. Des travaux sur le profil de taille de l'ADNcir sont en cours pour définir des approches de q-PCR et de séquençage massif en vue de son inclusion dans un test sanguin de dépistage du cancer.

\section{Un test fondé sur l'ADN circulant mitochondrial : le MiTest}

La quantité d'ADNcir dans le sang peut être un facteur discriminatif entre sujets sains et patients atteints de CCR [22], avec une AUC de 0,91 . La quantification et l'association de l'ADN mitochondrial circulant et nucléaire circulant permettent une telle distinction [52]. Nous avons déterminé un indice fondé sur la détection de séquences particulières dans le génome nucléaire et mitochondrial qui, appliqué à des surnageants de cultures cellulaires, montre une différence significative entre lignées cellulaires normales et cancéreuses. Dans le plasma, cet indice a une valeur statistiquement plus faible chez les patients atteints de cancer colorectal que chez les sujets sains. La mesure de cet indice, qui constitue ce que nous avons nommé le Mitest, constitue donc un test de dépistage puissant pour la détection précoce du cancer. Des études sont en cours afin de combiner ce nouveau marqueur avec d'autres paramètres pour augmenter le potentiel discriminant du test au sein de larges cohortes de patients et d'individus sains.

\section{Conclusion}

La biopsie liquide présente des avantages prometteurs pour le dépistage du cancer: sa nature minimalement invasive, sans le délai, le coût et les risques associés à la biopsie tissulaire, ainsi que la possibilité de détection à un stade précoce avant la détectabilité radiologique. Plusieurs biomarqueurs circulants sont actuellement évalués pour être validés en ce qui concerne leur utilité clinique (I'ADN circulant, les cellules tumorales circulantes, les microARN circulants et autres) pour développer des tests de détection précoce du cancer. Les exosomes, qui contiennent des acides nucléiques et des protéines, pourraient également être une source de multiples marqueurs de malignité dont l'analyse pourrait être prometteuse pour le développement de méthodes de dépistage [53]. Mais peu de ces biomarqueurs ont été à ce jour validés pour une utilisation clinique. L'ADNcir d'origine nucléaire et mitochondriale semble avoir un avantage dans le dépistage du cancer comparativement à d'autres biomarqueurs en montrant une meilleure efficacité et semble posséder les caractéristiques pour que sa détection soit mise en œuvre plus rapidement.

Il est à craindre ou à souhaiter que l'utilisation, à travers le monde, d'un test de dépistage se distingue de deux façons : (1) qu'il soit approuvé par les administrations de santé publique et remboursé, à la suite d'une étude statistique longue et rigoureuse, et (2) qu'il soit mis à disposition individuellement (par exemple, test de grossesse) avec un niveau de performance ou d'évaluation modéré et qu'il soit proposé dans un proche futur. La standardisation des paramètres pré-analytiques et une meilleure connaissance de l'origine et de la structure exacte de l'ADNcir constitueraient l'étape supplémentaire de sa mise en œuvre. L'avancement des méthodes sophistiquées de q-PCR ou de séquençage Next-Gen améliorera inévitablement la fiabilité de la performance analytique des futurs tests. Comme indiqué par Dennis Lo de I'Université de Hong Kong: «Brique par brique, nous sommes en train de mettre en place cette technologie ». À l'avenir, il semble, au vu des résultats prometteurs, que les biopsies liquides pourraient ajouter une nouvelle dimension au rôle du médecin eu égard au dépistage et au diagnostic. Pour le moins, l'étude de l'évaluation de la performance de dépistage de la biopsie liquide en tandem avec d'autres tests, comme l'IRM, semble justifiée. Par ailleurs, il est envisageable que des tests de biopsie liquide puissent être utilisés pour dépister le cancer à un stade précoce chez les individus à haut risque, tels que ceux atteints de cancers familiaux. Ainsi, il est crucial de poursuivre 
l'étude de ces biomarqueurs émergents. L'utilisation combinée de plusieurs marqueurs de l'ADN circulant ou bien de biomarqueurs conventionnels apparait être un moyen pour augmenter la sensibilité et la spécificité des tests et donc obtenir des résultats plus significatifs dans la détection précoce des cancers. Les années à venir semblent être excitantes alors que le «sacré graal » en cancérologie que constitue le dépistage universel apparaît être désormais accessible. $\diamond$

\section{SUMMARY}

\section{Liquid biopsy: a possible approach for cancer screening}

Liquid biopsy has emerged as a promising avenue for cancer screening. Several circulating biomarkers such as circulating DNA, circulating tumor cells, circulating microRNAs and others have shown promise for theragnostics and patient's monitoring. Early detection may help reduce cancer-related mortality and increase overall patient survival. Most cancer types lack specific biomarkers and despite intensive efforts in this area, the development of effective clinical screening techniques has been limited. The noninvasive nature of liquid biopsy represents an advantage over other approaches to define cancer biomarkers, particularly for the development of cancer screening tests. This review presents the various studies based on the analysis of liquid biopsy aiming to develop tests for cancer screening and early detection. So far, no test developed from liquid biopsy proves to be both specific and sensitive enough to be used as a universal screening test. However, the potential of this new approach appears more and more credible, given the recent developments of sophisticated multi-parametric methods. $\diamond$

\section{REMERCIEMENTS}

Nous remercions Safia El Messaoudi, Sandrine Bonizec et Amaelle Otandault pour leur aide. Nous remercions également Marc Ychou, Denis Pezet et Muriel Mathonnet pour avoir fourni les échantillons sanguins. Ce travail a été soutenu par l'Inserm, Lilly (France) et la bourse «INCa-DGOS-Inserm 6045 » du SIRIC de Montpellier, France.

\section{LIENS D'INTÉRÊT}

A.R. Thierry possède des actions de DiaDx SAS. R. Tanos déclare n'avoir aucun lien d'intérêt concernant les données publiées dans cet article.

\section{RÉFÉRENCES}

1. Maxim LD, Niebo R, Utell MJ. Screening tests: a review with examples. Inhal Toxicol $2014 ; 26: 811-$ 28.

2. Wilson JMG, Jungner G. Principles and practice of screening for disease. Geneva : World Health Organisation, $1968: 164 \mathrm{p}$.

3. Andermann A, Blancquaert I, Beauchamp S, et al. Revisiting Wilson and Jungner in the genomic age: a review of screening criteria over the past 40 years. Bull World Health Organ $2008 ; 86: 317-$ 19.

4. Hajian-Tilaki K. Receiver operating characteristic (ROC) curve analysis for medical diagnostic test evaluation. Casp J Intern Med $2013 ; 4: 627-35$

5. Mayrand MH, Duarte-Franco $\varepsilon$, Rodrigues I, et al. Human papillomavirus DNA versus Papanicolaou screening tests for cervical cancer. N Engl J Med 2007 ; 357 : 1579-88.

6. Stieber P, Nagel D, Blankenburg I, et al. Diagnostic efficacy of CA $15-3$ and CEA in the early detection of metastatic breast cancer: a retrospective analysis of kinetics on 743 breast cancer patients. Clin Chim Acta 2015 ; 448 : 228-31.

7. Catalona WJ, Smith DS, Ratliff TL, et al. Measurement of prostate-specific antigen in serum as a screening test for prostate cancer. N Engl J Med 1991 ; 324 : 1156-61.

8. Andriole GL, Crawford ED, Grubb RL, et al. Mortality results from a randomized prostate-cancer screening trial. $N$ Engl J Med 2009 ; 360 : 1310-19.

9. Schröder FH, Hugosson J, Roobol MJ, et al. Screening and prostate-cancer mortality in a randomized European study. N Engl J Med 2009 ; 360 : 1320-28.
10. Tsodikov A, Gulati R, Heijnsdijk EAM, et al. Reconciling the effects of screening on prostate cancer mortality in the ERSPC and PLCO trials. Ann Intern Med 2017; 167 : 449-55.

11. Chen JS, Chen KT, Fan WC, et al. Combined analysis of survivin autoantibody and carcinoembryonic antigen biomarkers for improved detection of colorectal cancer. Clin Chem Lab Med 2010 ; 48 : 719-25.

12. Song LL, Li YM. Current noninvasive tests for colorectal cancer screening: an overview of colorectal cancer screening tests. World J Gastrointest Oncol $2016 ; 8: 793-800$

13. Dhaliwal A, Vlachostergios PJ, Oikonomou KG, et al. Fecal DNA testing for colorectal cancer screening: molecular targets and perspectives. World J Gastrointest Oncol $2015 ; 7$ : 178-83.

14. Imperiale TF, Ransohoff DF, Itzkowitz SH, et al. Fecal DNA versus fecal occult blood for colorectal-cancer screening in an average-risk population. $N$ Engl J Med $2004 ; 351: 2704-14$.

15. Imperiale TF, Ransohoff DF, Itzkowitz SH, et al. Multitarget stool DNA testing for colorectal-cancer screening. N Engl J Med 2014 ; 370 : 1287-97.

16. $\mathrm{NCl}$ dictionary of cancer terms Natl Cancer Inst nd. https://www.cancer.gov/ publications/dictionaries/cancer-terms

17. Mandel $P$, Metais $P$. Les acides nucléiques du plasma sanguin chez l'homme. CR Seances Soc Biol Fil 1948 ; 142 : 241-3.

18. Stroun M, Anker P, Lyautey J, et al. Isolation and characterization of DNA from the plasma of cancer patients. Eur J Cancer Clin Oncol 1987 ; 23 : 707-12.

19. Thierry AR, Messaoudi S El, Gahan PB, et al. Origins, structures, and functions of circulating DNA in oncology. Cancer Metastasis Rev 2016; 35 : 347-76.

20. Ilie M, Hofman V, Long $\varepsilon$, et al. Current challenges for detection of circulating tumor cells and cell-free circulating nucleic acids, and their characterization in non-small cell lung carcinoma patients. What is the best blood substrate for personalized medicine? Ann Transl Med 2014 ; 2 : 107.

21. Wan JCM, Massie C, Garcia-Corbacho J, et al. Liquid biopsies come of age: towards implementation of circulating tumour DNA. Nat Rev Cancer 2017 ; $17: 223-38$.

22. Mouliere F, Messaoudi S El, Pang D, et al. Multi-marker analysis of circulating cell-free DNA toward personalized medicine for colorectal cancer. Mol Oncol $2014 ; 8$ : 927-41.

23. Church TR, Wandell M, Lofton-Day C, et al. Prospective evaluation of methylated SEPT9 in plasma for detection of asymptomatic colorectal cancer. Gut $2014 ; 63: 317-25$.

24. Lamb YN, Dhillon S. Epi proColon ${ }^{\circledR} 2.0$ CE: a blood-based screening test for colorectal cancer. Mol Diagn Ther 2017 ; 21 : 225-32.

25. Rasmussen SL, Krarup HB, Sunesen KG, et al. Hypermethylated DNA, a circulating biomarker for colorectal cancer detection. PLoS One 2017 ; 12 : e0180809.

26. Rahier JF, Druez A, Faugeras L, et al. Circulating nucleosomes as new bloodbased biomarkers for detection of colorectal cancer. Clin Epigenetics 2017 ; $9: 53$.

27. Jiang T, Zhai C, Su C, et al. The diagnostic value of circulating cell free DNA quantification in non-small cell lung cancer: a systematic review with meta-analysis. Lung Cancer 2016; $100: 63-70$.

28. Lin Z, Neiswender J, Fang B, et al. Value of circulating cell-free DNA analysis as a diagnostic tool for breast cancer: a meta-analysis. Oncotarget 2017 ; $8: 26625-36$.

29. Zhou Q, Li W, Leng B, et al. Circulating cell free DNA as the diagnostic marker for ovarian cancer: a systematic review and meta-analysis. PLoS One 2016 ; $11:$ e 0155495 .

30. Hosgood HD, Liu CS, Rothman N, et al. Mitochondrial DNA copy number and lung cancer risk in a prospective cohort study. Carcinogenesis $2010 ; 31$ : 847-49.

31. Thyagarajan B, Wang R, Barcelo $H$, et al. Mitochondrial copy number is associated with colorectal cancer risk. Cancer Epidemiol Prev Biomarkers $2012 ; 21: 1574-81$.

32. Ashworth TR. A case of cancer in which cells similar to those in the tumours were seen in the blood after death. Australasian Med J 1869 ; 14 : 146-7.

33. Tanaka F, Yoneda K, Kondo N, et al. Circulating tumor cell as a diagnostic marker in primary lung cancer. Clin Cancer Res $2009 ; 15: 6980-86$.

34. Fiorelli A, Accardo M, Carelli $\varepsilon$, et al. Circulating tumor cells in diagnosing lung cancer: clinical and morphologic analysis. Ann Thorac Surg 2015; 99 : 1899-905.

35. Ilie M, Hofman V, Long-Mira $\varepsilon$, et al. Sentinel circulating tumor cells allow early diagnosis of lung cancer in patients with chronic obstructive pulmonary disease. PLoS One 2014 ; 9 : el11597. 


\section{RÉFÉRENCES}

36. Wang $\mathrm{L}$, Wu C, piao $\mathrm{L}$, et al. Clinical significance of folate receptor-positive circulating tumor cells detected by ligand-targeted polymerase chain reaction in lung cancer. J Cancer $2017 ; 8$ : 104-10.

37. Diaz LA, Bardelli A. Liquid biopsies: genotyping circulating tumor DNA. J Clin Oncol 2014 ; 32 : 579 86.

38. Freidin MB, Freydina D V, Leung M, et al. Circulating tumor DNA outperforms circulating tumor cells for KRAS mutation detection in thoracic malignancies. Clin Chem $2015 ; 61$ : 1299-304.

39. Alix-Panabières C, Pantel K. Characterization of single circulating tumor cells. FEBS Lett 2017 ; $591: 2241-50$.

40. Roth C, Rack B, Müller V, et al. Circulating microRNAs as blood-based markers for patients with primary and metastatic breast cancer. Breast Cancer Res $2010 ; 12:$ R90.

41. Kodahl AR, Lyng MB, Binder $H$, et al. Novel circulating microRNA signature as a potential noninvasive multi-marker test in $\varepsilon R$-positive early-stage breast cancer: a case control study. Mol Oncol $2014 ; 8: 874-83$.

42. Tomasetti $M$, Amati $M$, Neuzil J, et al. Circulating epigenetic biomarkers in lung malignancies: from early diagnosis to therapy. Lung Cancer 2017 ; $107: 65-72$.

43. Adams DL, Adams DK, Alpaugh RK, et al. Circulating cancer-associated macrophage-like cells differentiate malignant breast cancer and benign breast conditions. Cancer Epidemiol Prev Biomarkers $2016 ; 25: 1037-42$.

44. Gonzalez RM, Daly DS, Tan R, et al. Plasma biomarker profiles differ depending on breast cancer subtype but RANTES is consistently increased. Cancer Epidemiol Biomarkers Prev $2011 ; 20: 1543$ 51.

45. Sol N, Wurdinger T. Platelet RNA signatures for the detection of cancer. Cancer Metastasis Rev $2017 ; 1-10$.
46. Phallen J, Sausen M, Adleff V, et al. Direct detection of early-stage cancers using circulating tumor DNA. Sci Transl Med 2017 ; 9 : eaan2415.

47. Fernandez-Cuesta L, Perdomo S, Avogbe PH, et al. Identification of circulating tumor DNA for the early detection of small-cell lung cancer. EBioMedicine 2016 ; 10 : 117-23.

48. Cohen JD, Li L, Wang Y, et al. Detection and localization of surgically resectable cancers with a multi-analyte blood test. Science 2018 ; eaar3247.

49. Chan KCA, Woo JKS, King A, et al. Analysis of plasma Epstein-Barr virus DNA to screen for nasopharyngeal cancer. N Engl J Med 2017 ; 377 : 513-22.

50. Mouliere F, Robert B, Peyrotte $\varepsilon A$, et al. High fragmentation characterizes tumour-derived circulating DNA. PLoS One $2011 ; 6$ : e23418.

51. Leszinski G, Lehner J, Gezer U, et al. Increased DNA integrity in colorectal cancer. In Vivo (Brooklyn) $2014 ; 28: 299-303$.

52. Thierry A, Messaoudi SE. Methods for screening a subject for cancer. W02016/063122.

53. Wang M, Ji S, Shao G, et al. Effect of exosome biomarkers for diagnosis and prognosis of breast cancer patients. Clin Transl Oncol 2017 ; 1-6.

54. Loeb $S$, Catalona WJ. The prostate health index: a new test for the detection of prostate cancer. Ther Adv Urol $2014 ; 6: 74-7$.

55. Jordan B. Dépister les cancers asymptotiques? Med Sci (Paris) 2018 ; 34 363-5.

\section{TIRÉS À PART}

A.R. Thierry

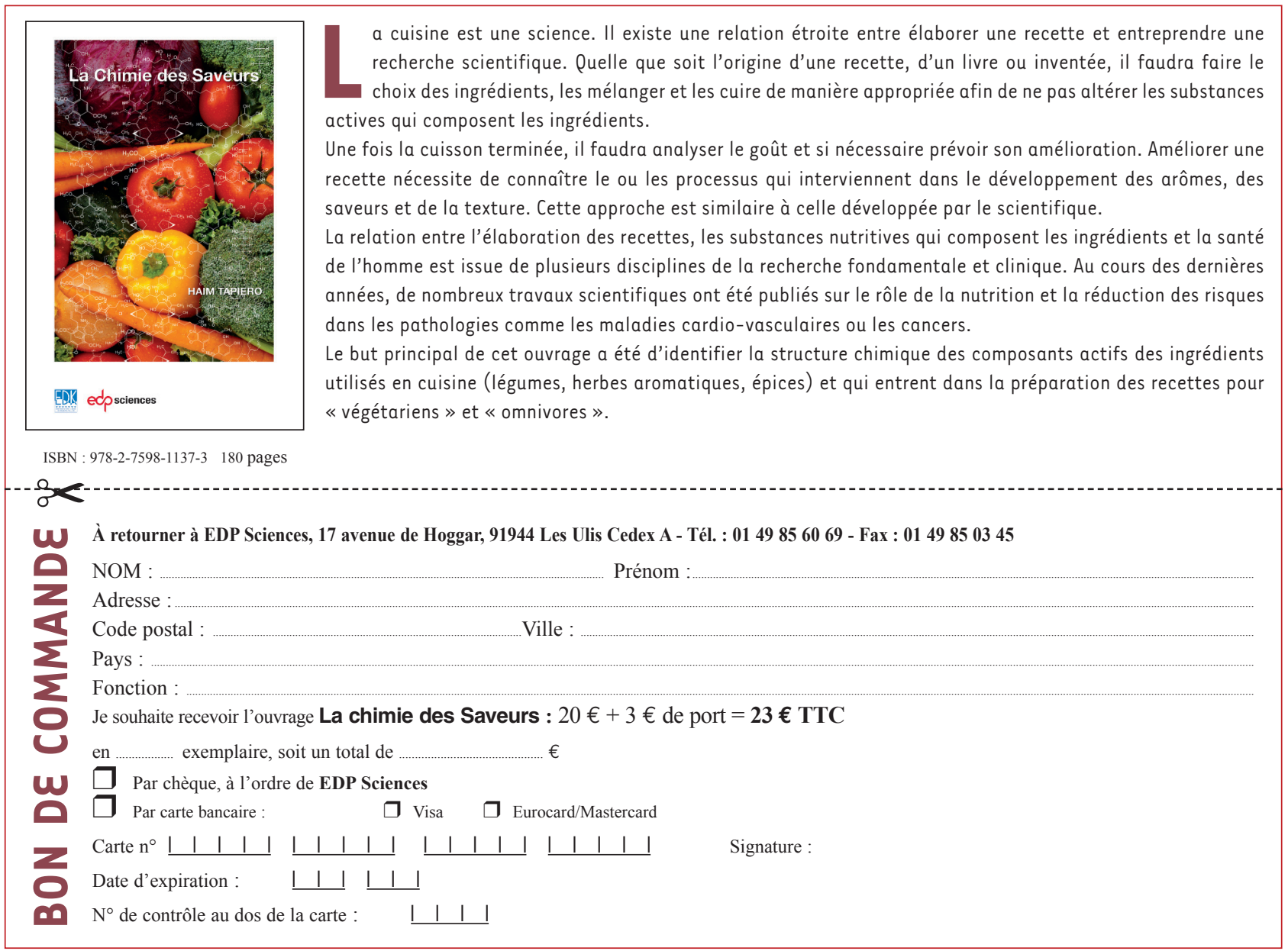

